\title{
Article
}

\section{Healthcare Utilisation, Morbidities and Alcohol Use Monitoring Prior to Alcoholic Psychosis Diagnosis}

Otete, Harmony, Fleming, M Kate, West, Joe and Orton, Elizabeth Available at http://clok.uclan.ac.uk/25573/

Otete, Harmony ORCID: 0000-0003-2467-2605, Fleming, M Kate, West, Joe and Orton, Elizabeth (2019) Healthcare Utilisation, Morbidities and Alcohol Use Monitoring Prior to Alcoholic Psychosis Diagnosis. Alcohol and Alcoholism, 54 (2). pp. 131-138. ISSN 0735-0414

It is advisable to refer to the publisher's version if you intend to cite from the work. http://dx.doi.org/10.1093/alcalc/agy085

For more information about UCLan's research in this area go to http://www.uclan.ac.uk/researchgroups/ and search for <name of research Group>.

For information about Research generally at UCLan please go to http://www.uclan.ac.uk/research/

All outputs in CLoK are protected by Intellectual Property Rights law, including Copyright law. Copyright, IPR and Moral Rights for the works on this site are retained by the individual authors and/or other copyright owners. Terms and conditions for use of this material are defined in the policies page.

\section{CLoK}

Central Lancashire online Knowledge www.clok.uclan.ac.uk

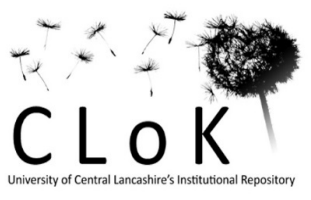




\section{Healthcare utilisation, morbidities and alcohol use monitoring prior to alcoholic psychosis diagnosis.}

Otete Harmony, Assistant Professor ${ }^{1,2}$

Fleming M Kate, Associate Professor ${ }^{3}$

West Joe, Professor ${ }^{1}$

Orton Elizabeth, Associate Professor ${ }^{4}$

\section{Affiliations}

${ }^{1}$ Division of Epidemiology and Public health, School of Medicine, University of Nottingham, City Hospital Campus, Nottingham, NG5 1PB, United Kingdom.

${ }^{2}$ School of Medicine, University of Central Lancashire, Preston, PR1 2HE, United Kingdom

${ }^{3}$ Department of Public health and Policy, University of Liverpool, Liverpool, L69 3GB, United Kingdom.

${ }^{4}$ Division of Primary care, School of Medicine, University of Nottingham, University Park, Nottingham, NG7 2RD, United kingdom.

Correspondence to: Harmony E Otete, Email: hotete@uclan.ac.uk

Declaration of interest: There are no conflicts of interest to declare for any of the authors. 


\section{Abstract}

Aims: This study aimed to describe healthcare utilisation, morbidities and monitoring of alcohol use in patients prior to a diagnosis of alcoholic psychosis in order to inform the early identification of patients at risk.

Method: Using linked general practice and hospitalisation data in England (April 1997 to June 2014) we identified 1,731 individuals ( $\geq 18$ years) with a clinical recorded diagnosis of alcoholic psychosis and 17,310 matched controls without the disorder. We examined all prior general practitioner (family doctor) visits, hospitalisations, medically-recorded morbidities, alcohol assessment/interventions records. Poisson regression models were used to compare rates of healthcare utilisation in people with alcoholic psychosis to those without. Logistic regression models were used to evaluate the association between alcoholic psychosis and prior morbidities.

Results: Patients with alcoholic psychosis showed increased levels of healthcare utilisation at least 5 years prior to their diagnosis. The most common reasons for prior healthcare visits were seizures and injuries and there was more than 4-fold higher rate of seizures, unintentional injuries and self-harm incidents among these patients up to 10 years prior to diagnosis, compared to the control population. A high proportion (78\%) of patients had their alcohol consumption recorded, 50\% had a record of heavy drinking but only 1 in 5 had any evidence of receiving an alcohol-related intervention.

Conclusion: Patients present more often with seizures and injuries than the general population several years prior to a diagnosis of alcoholic psychosis. These visits represent opportunities for preventive action and imply that we may be missing opportunities to intervene. 


\section{Summary}

Several years prior to a diagnosis of alcoholic psychosis, patients present more often with seizures and injuries (falls, fractures, assaults and self-harm) than the general population. Targeting interventions at such patients could be a more sensitive way of identifying heavy drinkers that are more likely to develop alcoholic psychosis. 


\section{Introduction}

Alcoholic psychosis is a mental and behavioural disorder resulting from chronic alcoholism (1). Although distinct from Schizophrenia, the core features of the syndrome include delusions; hallucinations, usually in the form of derogatory and threatening voices; mood disorders, impaired thought process and inappropriate behaviour (2). The lifetime prevalence of alcoholic psychosis has been estimated to approximate $0.5 \%-1.8 \%(3,4)$. However, in individuals who develop the disorder, there are serious health risks including an increased risk of suicidal behaviour with or without depression, and a rehospitalisation rate of up to $60 \%$ (2-5). Mental and behavioural disorders due to alcohol use accounted for over 200,000 hospital admissions in 2012 in the UK and remain among the top three causes of alcohol related morbidity in the UK (6). Currently, there is no evidence-based treatment available for Alcoholic psychosis and so preventing chronic alcohol use in the first place is the main option currently for interrupting the pathway leading to the disease.

Extensive evidence both in the UK and globally show that alcohol screening and brief interventions can effectively interrupt the course of alcohol harm by first, identifying people who consume alcohol at levels that put their health at risk, and motivating a positive change in drinking behaviour to lower risk levels (7-10). The use of alcohol screening combined with information on early indicators of alcohol psychosis could aid earlier identification and better targeting of at-risk individuals, if these indicators are known by practitioners. However, to date, there is limited research into the pathway leading to a diagnosis of alcoholic psychosis. More specifically, there are very few published studies describing healthcare utilisation prior to alcoholic psychosis with even fewer studies describing what patients present with prior to the disease.

With access to linked primary care and hospitalisation data in the UK, there is an opportunity to study healthcare use and the clinical trajectory leading up to a diagnosis with alcoholic psychosis in order to inform early detection and prevention strategies. We have therefore 
carried out a retrospective study on individuals diagnosed with alcoholic psychosis using these linked data. We specifically aimed to describe patterns of help-seeking in primary and secondary care and to identify the other alcohol related morbidities patient present with prior to diagnosis of alcoholic psychosis. To understand the current practice relating to alcohol interventions and to further inform alcohol harm preventive strategies, we quantified the number of patients who received an alcohol assessment or alcohol brief intervention prior to alcoholic psychosis diagnosis. 


\section{Methods}

The linked primary care and hospitalisation data were obtained from the Clinical Practice Research datalink(CPRD) and Hospital Episodes Statistics(HES) respectively.

General Practitioners (family doctors) in the UK are the gatekeepers of most types of secondary healthcare and nearly $98 \%$ of the total UK population are registered with a general practice. The CPRD database holds medical records from over 600 UK general practices serving approximately $6 \%$ of the total UK population and is nationally representative (11). Data recorded within the CPRD include patient demography, clinical diagnoses coded using Read codes (based on the International Classification of Diseases version 10 ICD-10), patient prescriptions, test results, behavioural habits such as alcohol use and smoking status, and behavioural interventions. These data are recorded prospectively by General Practitioners during primary care consultations or following correspondence regarding secondary care treatment, and have been extensively validated for research purposes (12).

More than $50 \%$ of the patients in CPRD are linkable to hospitalisation data which is held within the Hospital Episodes Statistics (HES) database. HES captures information on all inpatient hospital admissions in England and includes hospital diagnoses coded using the ICD-10. For this study, we restricted our source population to only those patients that are linkable across CPRD and HES to allow us obtain a comprehensive history of the health service utilisation of each patient. Linked hospitalisation data were available from 1997 to 2014. Approval for use of these linked data was given by the Scientific and Ethical Committee of the CPRD (15_073RAR).

\section{Study population}

Using a list of Read and ICD 10 diagnostic codes, we identified all patients with a diagnosis of alcoholic psychosis between April 1997 and June 2014. To avoid being restrictive, we also included patients with other alcohol-related syndromes associated with psychotic 
features such as alcoholic paranoia, alcoholic dementia, and alcoholic hallucinosis. Table 1 shows the list of Read and ICD-10 diagnosis codes used.

Table 1: Read and ICD 10 diagnosis used for identifying study population

\begin{tabular}{|c|c|}
\hline Codes & Diagnosis \\
\hline \multicolumn{2}{|l|}{ ICD 10} \\
\hline $\begin{array}{l}\text { F10.5 } \\
\text { F10.7 } \\
\text { F10.8 } \\
\text { F10.9 }\end{array}$ & $\begin{array}{l}\text { Mental and behavioural disorders due to use of alcohol, psychotic disorder } \\
\text { Mental and behavioural disorders due to use of alcohol, residual and late-onset } \\
\text { psychotic disorder } \\
\text { Mental and behavioural disorders due to use of alcohol, other mental and } \\
\text { behavioural disorders } \\
\text { Mental and behavioural disorders due to use of alcohol, unspecified mental and } \\
\text { behavioural disorder }\end{array}$ \\
\hline \multicolumn{2}{|l|}{ Read Codes } \\
\hline Eu10513 & Alcoholic paranoia \\
\hline E011000 & Korsakov's alcoholic psychosis \\
\hline E015.00 & Alcoholic paranoia \\
\hline Eu10514 & Alcoholic psychosis NOS \\
\hline E011100 & Korsakov's alcoholic psychosis with peripheral neuritis \\
\hline E01z.00 & Alcoholic psychosis NOS \\
\hline Eu10711 & Alcoholic dementia NOS \\
\hline E01y.00 & Other alcoholic psychosis \\
\hline Eu10511 & Alcoholic hallucinosis \\
\hline E01..00 & Alcoholic psychoses \\
\hline E01yz00 & Other alcoholic psychosis NOS \\
\hline 1B1c.00 & Alcohol induced hallucinations \\
\hline Eu10500 & Mental \& behavioural disorder due to use alcohol: psychotic disorder \\
\hline E011200 & Wernicke-Korsakov syndrome \\
\hline Eu10611 & Korsakov's psychosis, alcohol induced \\
\hline E012.00 & Other alcoholic dementia \\
\hline
\end{tabular}

Each identified patient was assigned an index date of diagnosis which was the date of the first record of any of the above conditions in either their primary care (CPRD) or secondary care(HES) record. We excluded patients whose diagnosis date fell within 1 year of registration with a general practice (to exclude prevalent cases) or patients with invalid or missing diagnosis dates. Further exclusions were patients less than 18 years old at diagnosis, patients flagged as unacceptable for research as per CPRD quality rules (which is 
usually an indicator of patients with incomplete/inconsistent follow-up data), and those diagnosed outside the up-to-research-standard time periods as per CPRD quality rules.

Comparison group

For each identified patient, we selected up to 10 general population controls matched by general practice, sex and age (within 5 years). Each identified control was assigned an index date, which was a randomly generated date from 1 year after the start of the linked dataset (1997) up to the date they left their general practice or died. We excluded controls who had prior history of any type of psychosis before their pseudo-diagnosis date; this allowed the exclusion of patients with psychosis that may be alcohol related but are not recorded as such.

\section{Outcome measures}

\section{Healthcare utilisation}

Our assessment of healthcare utilisation was as we have previously reported (13). Briefly, we extracted data on all primary care consultations and in-patient hospitalisations prior to the index date for the alcoholic psychosis cohort and for the comparison group.

\section{Prior morbidities of interest}

Morbidities assessed are those known to be associated with alcohol use which could potentially serve as indicators of alcohol harm. Public Health England has produced a national guide on alcohol-attributable burden which contains a summary of these morbidities(6). The guide, which has been validated against other international sources, includes over 30 different morbidities broadly categorised in two groups: 1) alcohol specific conditions (alcoholic liver damage, alcohol-induced pseudo-cushing's syndrome, accidental 
poisoning by methanol or ethanol, alcoholic cardiomyopathy, alcohol-induced chronic pancreatitis, alcoholic gastritis, acute alcoholic intoxication, alcoholic myopathy, cerebral degeneration due to alcoholism, alcoholic encephalopathy, alcoholic polyneuropathy) and 2) alcohol related conditions (oral cancers, oesophageal cancer, colorectal cancer, breast cancer, diabetes mellitus, epilepsy, hypertensive disease, ischaemic heart disease, haemorrhagic stroke, ischaemic stroke, road traffic injuries, falls, drowning, poisonings, selfharm injuries, and interpersonal violence).

We searched patients' primary and secondary care records using relevant Read and ICD-10 codes to identify patients with these morbidities. Where a patient had multiple consultations or hospital admissions with a specific code, only the earliest record was kept for this analysis. Multiple records of injuries were treated differently as injuries differ from chronic conditions whereby people can have multiple acute injuries over time. Instead of the earliest record, we kept records of all new injuries for our analysis. A new injury was defined as a diagnostic code entered 30days (for all injury types except fractures) or 100 days (for fractures) after an initial code for the same injury, for example if a patient had a first record of a fall and another record $>30$ days later, we kept both records as two different fall injuries. These time windows were chosen through examining of the distribution of medical codes after an initial injury event which showed that codes entered into the medical records due to repeat consultations for the same injury event levelled off around 30 days for most injures and around 90 days for fractures.

Where patients had records of different morbidities entered on the same date, for example if an individual received a diagnosis code for hypertension and was also diagnosed with diabetes on the same date, both records of unique diseases were included. All alcohol related diseases were further categorised according to ICD chapter headings (malignant neoplasms, diabetes, diseases of the nervous system, cardiovascular diseases, digestive diseases, and injuries) for clarity of presentation. Injuries were categorised as either intentional (e.g. self-harm) or unintentional (e.g. falls), and where intent was not specified the 
injury event was classed as unintentional. We defined four exposure periods prior to patients index date to assess morbidities: within 12 months of diagnosis, 13 months-2 years prior to diagnosis, 3-5 years prior to diagnosis and 6-10 years prior to diagnosis.

\section{Recording of alcohol consumption behaviour and related interventions}

All alcohol records within the CPRD database were extracted for each patient. These records are typically either numerical (units of alcohol consumed per week by a patient) or clinically coded (Read codes which reflect various levels of drinking or interventions). Using the highest drinking record derived for each patient based on the available unit or Read coded information, patient alcohol use was categorised into three levels: "never drinkers", "moderate drinkers", "harmful or hazardous drinkers". Our previous study (14) has shown that it is not always possible to assign an alcohol consumption category to all patients with alcohol records (those affected are usually patients with a record of an alcohol assessment test such as the AUDIT without any explicit consumption record). In such cases an 'unclear' consumption status was assigned. A separate category was created for those who had records of Read codes for alcohol brief intervention or alcohol related referrals irrespective of which drinking category they were in.

\section{Other covariates}

We extracted data on patient sex, age and smoking history from CPRD as potential confounders. Age was calculated as age at diagnosis of alcoholic psychosis (or at pseudodiagnosis for the population controls) and categorized into five age bands of $18-44,45-54$, 55-64, 65-74 and $\geq 75$ years. Smoking status was categorised as current smoker, exsmoker and non-smoker using the most recent smoking data recorded before a patient's 
diagnosis or pseudo-diagnosis date, and a $4^{\text {th }}$ category "missing" was included for those without any smoking information.

\section{Statistical analysis}

Descriptive statistics were calculated for the study cohort and the control population. Healthcare utilisation was described at two levels; the overall healthcare utilisation rate for the study period and the yearly utilisation rate over the 10-year period before alcoholic psychosis diagnosis. The difference in utilisation rates between patients with alcoholic psychosis and the control population was estimated as the absolute difference in rates between both groups. Poisson regression models were used to generate rate ratios and 95\% Confidence Intervals $(\mathrm{Cl})$. Likelihood ratio tests were used to test for interaction between sex/or age and healthcare utilisation with the significance level defined at $p<0.05$.

The prevalence of a specific morbidity was calculated as the number of patients ever diagnosed as the numerator and the number of patients with incident alcoholic psychosis or matched controls as denominator. Logistic regression was used to estimate Odds Ratios (ORs) for the association between alcoholic psychosis and each specific morbidity for the entire study period and for the four predefined exposure periods (within 12 months of diagnosis, 13 months-2 years prior to diagnosis, 3-5 years prior to diagnosis and 6-10 years prior to diagnosis). Only people considered to be at risk (based on length of follow-up) were considered to estimate the prevalence or OR's in each study period. Effect modification by age and sex was tested for via stratified analyses and the fitting of interaction terms in logistic regression models; the significance of models was tested using likelihood ratio tests (LRTs), with $\mathrm{p}<0.05$ considered significant.

To quantify the cases who had been previously given an alcohol assessment or alcohol intervention, numbers and proportions were calculated for each alcohol consumption category ('never drinkers', 'moderate drinkers' or 'harmful/hazardous drinkers') and for 
patients with any record of a brief alcohol intervention/referral. All other patients without alcohol consumption, or intervention/referral records were labelled as 'no data available'.

Where possible, regression models were refitted applying the generalized estimating equation (GEE) approach with exchangeable correlation structure to test for heterogeneity within GP practices.

All statistical analyses were conducted using Stata statistical software version 13.0 (StataCorp Inc., College Station, TX, USA). 


\section{Results}

We included 1,731 patients with alcoholic psychosis and 17,310 matched control patients from 338 English GP practices (Table 1). The median observation time before diagnosis /pseudo-diagnosis was 5.5 years for the alcoholic psychosis cohort and 4.4 years for the control group. The mean (S.D) age at alcoholic psychosis diagnosis was 53.9 years (S.D 17.3). Among patients with alcoholic psychosis, there were more men and more current smokers (Table 2).

\section{Healthcare utilisation}

During the study period, the rate of primary care consultation among alcoholic psychosis patients was approximately 10 consultations per year. This corresponded to a $53 \%$ higher consultation rate [Rate ratio $1.53, \mathrm{Cl} 1.52$ to 1.54 ] or an average excess of 3 more consultations per year in patients with alcoholic psychosis compared to the control population. The greatest excesses in primary care use were found in the years closest to alcoholic psychosis diagnosis (Table 3). Being male was associated with greater excesses in consulting with primary care when compared to the control population ( $P$ value for likelihood ratio test for sex interaction $<0.0001)$. No significant interaction was found between age and primary care consultation rates in people with alcoholic psychosis.

Patterns of hospitalisation rates were also similar to that of primary care consultations. Prediagnosis hospitalisations were more than two-fold greater in patients with alcoholic psychosis than in the control population (IRR 2.4, 95\% Cl 2.3-2.5) (Table 4). Again, though most marked in the years before diagnosis, the difference in hospitalisation rates was apparent as early as up to 8 years before the diagnosis of alcoholic psychosis. There was a slightly higher number of excess hospitalisations in men than women within three years before the diagnosis of alcoholic psychosis (Table 4), but earlier than that, the annual 
excesses were higher in women (likelihood ratio test for interaction between sex and rates of admission $\mathrm{p}=0.04$ ).

\section{Prior morbidities among patients}

A total of $66.8 \%$ of patients with alcoholic psychosis had presented with at least one alcohol attributable condition during a preceding primary care visit or hospitalisation, compared to $33.2 \%$ of the control population (Table 5). A higher proportion of patients presented with alcohol-related conditions compared to alcohol-specific conditions (63.8\% vs. $20.5 \%)$. Due to insufficient numbers in many disease categories, results for each alcohol specific condition are not presented. Among the alcohol-related morbidities, all except malignant neoplasms were statistically significantly more prevalent in the alcoholic psychosis cohort. However, the most common were unintentional injuries (41.1\%), intentional injuries (18.8\%), cardiovascular diseases (22.9\%) and epileptic seizures (7.7\%). Epileptic seizures (OR 7.1, $95 \% \mathrm{Cl}$ 5.6-9.0), unintentional injuries (OR 4.5, 95\% $\mathrm{Cl} 4.0-5.0$ ) and intentional injuries (OR $11.8,95 \% \mathrm{Cl} 9.8-14.1)$ showed the strongest association with later diagnosis of alcoholic psychosis. The calculation of OR's over time, showed that the association between injuries (unintentional and intentional), seizures and alcoholic psychosis persisted up to 10 years before diagnosis, while other disease associations did not(Figure 1).

\section{Alcohol records}

We found prior records of alcohol use status for $79.1 \%$ of patients with alcoholic psychosis and $65.4 \%$ of controls (Table 6 ). When stratified by levels of drinking, $50 \%$ of the alcoholic psychosis cohort had a harmful or hazardous drinking record prior to alcoholic psychosis diagnosis (Table 2). Analysis of the timing of records showed that most patients were recorded as heavy drinkers, on average, up to 5 years before the diagnosis of alcoholic psychosis. Men with alcoholic psychosis were more likely than women to have any alcohol consumption record [men adjusted OR 1.7, 95\% Cl 1.5-2.0; women adjusted OR 1.2, 95\% 
$\mathrm{Cl}$ 0.9-1.5; likelihood ratio test $P$ value for sex interaction=0.0012) (Table 6). We found no significant difference in the prevalence of records across age-groups (Likelihood ratio test $p$ value for age interaction 0.12 ).

A much lower proportion of patients were found to have prior records of interventions. We found records of an alcohol brief intervention or alcohol related referrals to other services for 1 in every 5 patients with alcoholic psychosis (Table 7). When results were stratified across age groups, significant differences were identified (likelihood ratio test $p$ value for age interaction= 0.02) with records showing patients aged 18-44 years [OR 4.3 95\% $\mathrm{Cl} 3.1-5.8$ ] were more likely to be offered alcohol brief interventions or referred on to other alcohol services compared to those who were older [OR for $45-54$ years 3.2, 95\% Cl 2.2-4.8; OR for $\geq 75$ years $2.4,95 \% \mathrm{Cl} 1.5-3.7]$ (Table 7 ). When results were stratified by sex, we found no difference between men and women (likelihood ratio test $p$ value for sex interaction $=0.06$ ). 


\section{Discussion}

\section{Summary of main findings}

This study has shown that prior to alcoholic psychosis diagnosis, patients have a high healthcare utilisation, of around $50 \%$ increase per annum compared with an age, sex, and general practice-matched control population. This excess in healthcare utilisation may be explained by a higher prevalence of alcohol related morbidities in patients, as approximately two-thirds of patients were seen for such morbidities compared to $33 \%$ in the control population. Of all assessed alcohol related morbidities, seizures and injuries (both intentional and unintentional) were more strongly associated with a later development of alcoholic psychosis. Assessing injuries in slightly more detail suggested that falls, assaults and selfharm were accountable for $>80 \%$ of the injury burden among patients.

In terms of how often alcohol assessments and interventions were offered to patients prior to alcoholic psychosis diagnosis, $79 \%$ of patients with alcoholic psychosis had previously had an alcohol assessment (based on records of alcohol status being available). However, it was only in 1 in 5 patients that interventions to reduce alcohol consumption were recorded. There were significant differences in alcohol records across subgroups such that men were more likely than women to have a record of their alcohol use in primary care, but not an alcohol related intervention. Younger age groups (18-44 years) were more likely to have records of interventions than those who were older.

\section{Strengths and limitations}

This is the first longitudinal study to comprehensively describe primary and secondary care use and alcohol-related morbidities among people who go on to develop alcoholic psychosis, compared with a representative general population control group, and therefore the first to provide insight into opportunities for earlier interventions. By using contemporary, nationally 
representative data collected prospectively during routine clinical care, our findings are generalizable to most current patients in England with clinically diagnosed alcoholic psychosis. Although we have used only patients from half of the practices contributing to CPRD (as these are the only practices where secondary care records are linkable), it has been suggested this is unlikely to affect generalisability as patients from linked CPRD practices are reasonably similar in terms of age and sex to those from non-linkable practices (15). The large size of the dataset has allowed us to generate robust estimates of healthcare use and of the associations between alcoholic psychosis and alcohol related morbidities. We have also been able to minimise recall bias inherent to retrospective studies, by virtue of the prospective nature that data in CPRD are recorded.

As with any study using routinely available data, an inherent potential weakness is the validity of the diagnostic data of each patient. CPRD has been previously validated for a wide range of diseases including psychosis, cardiovascular, digestive diseases and cancers. Validation studies on psychosis in primary care have however not focused specifically on the alcoholic psychosis subgroup (16). Although we do not believe there is any reason why the validity of this subgroup should be dissimilar from the rest of the psychosis population, we are still unable to assess nor guarantee the sensitivity of diagnostic codes such as "Korsakoff's alcoholic psychosis" and this may be a potential source of diagnostic inaccuracy in our study. Another potential limitation is that by defining interventions based on information recorded within patient records, we may have underestimated the true rate at which practitioners intervene for heavy drinking in the UK as there may be cases where brief advice to cut down on drinking is provided but not recorded as an intervention. Nevertheless, this underestimation would not explain the differences we have identified between men and women and across age-groups as there is not likely to be a bias in operation leading to increased /decreased recording of information influenced by age and gender. Insofar as part of the rationale of this study is to provide information that would improve the identification and treatment of people who drink heavily, we see this potential for underestimation due to 
lack of recording not as a study weakness, but rather as a pointer to a potential area for practitioners to address. It is worth noting that we have not assessed for changes in rates of intervening over time, so we are unable to highlight any change/improvement that has happened in recent years. We also cannot rule out the possibility that some of the higher prevalence of comorbidity in the alcoholic psychosis group may be due to better case finding prompted by higher patient contact with health services. Nevertheless, in injuries and seizures specifically, presentation is rather acute which suggests that the high frequency is more likely to be explained by a true increase in disease burden rather than opportunistic diagnostic probing. Our data showed that falls, fractures, assault and self-harm account for $>80 \%$ of the injury burden in the alcoholic psychosis cohort, nonetheless further work evaluating detailed dimensions of injury, such as mechanism, severity and location, may be useful to inform the development targeted interventions for patients.

\section{Comparison to previous literature}

In relation to previously published work, prior studies on healthcare use in people with clinically diagnosed alcoholic psychosis are scarce. However, our finding that there is a high burden of specific alcohol related comorbidities prior to alcoholic psychosis diagnosis is comparable to results in the study by Perala et al. Although Perala et al's study differs by not being population based, by assessing fewer comorbidities, and by selecting an alcohol dependent control population, they also found a higher rate of fractures [OR 5.13], epilepsy [OR 2.8] and arrhythmias [OR 3.43] among 38 cases of alcoholic psychosis compared to the control population (4).

Although not directly comparable, our previously published papers on people with alcoholic cirrhosis also found a high rate of healthcare utilisation for alcohol related morbidities among patients. However, by contrast, we found much stronger association for injuries (OR 5.7 vs. 4.0) and epileptic seizures (OR 7.1 vs 4.4) in people with psychosis than in alcoholic cirrhosis. 
Our results on the provision of alcohol treatment are also in agreement with other population-based research and reviews which report that the frequency of intervening for alcohol use among practitioners is low both in the UK and internationally (17-22). The reasons for this low uptake have ranged from practitioner to resource factors including insufficient time during consultations, inadequate training whereby practitioners do not feel competent to deal with alcohol related issues, lack of skills and lack of financial support from government policy (23).

\section{Conclusions and implications}

Overall, in this study we have found a higher rate of health service usage, higher incidence of injuries and seizures, and low level of recorded alcohol related interventions prior to alcoholic psychosis which have significant implications for preventing the disease and for healthcare professionals who take care of these patients.

Together, these findings support the need for practitioners to optimise the provision (and/or recording) of alcohol related advice and support in accordance with guidance from the National Alcohol strategy and the National Institute for Health and Care Excellence (NICE). Optimising recording and/or delivery of alcohol support would not only impact positively on patients at risk of alcoholic psychosis but is required to address concerns over the high level of avoidable alcohol related morbidity and mortality in the UK. Failure to do so could be detrimental and have knock-on effects on individuals, their families and carers, and healthcare services, whereby hospital and mental health services increasingly see patients with illnesses that could have been averted at a much earlier stage.

Based on the comorbidity results, perhaps, a closer review of people presenting with epileptic seizures and injuries (falls, fractures, assaults and self-harm) could be a more sensitive way of identifying harmful/hazardous drinkers that are more likely to develop alcoholic psychosis. When these patients are identified, evidence-based interventions can then be provided at an early stage when they are likely to be beneficial. 
1. Rehm J, Room R, Monteiro M, Gmel G, Graham K, Rehn N. Alcohol Use. In: Ezzati M, Lopez A, Rodgers A and Murray C. Eds. Comparative quantification of health Risks Volume 1. WHO:2004, pp 959-1108.

2. Soyka, M., Helten, B., Cleves, M. Schmidt P. High rehospitalization rate in alcoholinduced psychotic disorder. Eur Arch Psychiatry Clin Neurosci 2013; 263(4): 309313.

3. Jordaan GP, Warwick JM, Nel DG, Hewlett R, Emsley R. Alcohol-induced psychotic disorder: brain perfusion and psychopathology--before and after anti-psychotic treatment. Metab Brain Dis. 2012 Mar;27(1):67-77.

4. Perälä J, Kuoppasalmi K, Pirkola S, Härkänen T, Saarni S, Tuulio-Henriksson A, et al. Alcohol-induced psychotic disorder and delirium in the general population. $\mathrm{Br} \mathrm{J}$ Psychiatry. 2010 Sep 1;197(3):200-6.

5. Jordaan GP, Emsley R. Alcohol-induced psychotic disorder: a review. Metab Brain Dis. 2014 Jun;29(2):231-43.

6. Jones L, Bellis M. Updating England-Specific Alcohol-attributable Fractions. Centre for Public health: Liverpool John Moore University.

7. Fleming M, Manwell L. Brief Intervention in Primary Care settings: A primary Treatment Method for At-Risk, Problem and Dependent drinkers. Alcohol Res Health. $1999 ; 23(2): 128-37$.

8. NICE. Alcohol use disorders: preventing the development of hazardous and harmful drinking. UK; 2010. 
9. Babor T, Higgins-Biddle J. Brief intervention for harzadous and harmful drinking: A manual for use in primary care. [Internet]. World Health Organisation; 2001. Available from: http://whqlibdoc.who.int/hq/2001/who msd msb 01.6b.pdf

10. UK Government. The Government's Alcohol Strategy. 2012.

11. Clinical Practice Research Datalink [Internet]. 2012. Available from: http://www.cprd.com/intro.asp.

12. Walley T, Mantgani A. The UK General Practice Research Database. lancet. 350:1097-9.

13. Otete HE, Orton E, West J, Fleming KM. Sex and age differences in the early identification and treatment of alcohol use: a population based study of patients with alcoholic cirrhosis. Addiction. 2015; Aug 1. doi: 10.1111/add.13081

14. Otete HE, Orton E, Fleming KM, West J. Alcohol-attributable health care attendances up to 10 years prior to diagnosis of alcoholic cirrhosis: a population based case control study. Liver International 2015 Nov 12. doi: 10.1111/liv.13002.

15. Crooks CJ. Epidemiology of upper gastrointestinal bleeding. Ph.D. Thesis. University Of Nottingham: UK.; 2013.

16. Herrett E, Thomas S, Schoonen M, Smeeth L, and Hall A. Validation and validity of diagnoses in the General Practice Research Database: a systematic review. Br J Clin Pharmacol. 2010;69(1):4-14.

17. Cheeta, S., Drummond, C., Oyefeso, A., Phillips, T., Deluca, P., Perryman, K. and Coulton, S. (2008), Low identification of alcohol use disorders in general practice in England. Addiction, 103: 766-773. doi:10.1111/j.1360-0443.2008.02198.x

18. Kaner E, Heather N, Mcavoy B, Lock C, Gilvarry E. Intervention for excessive alcohol consumption in primary health care: attitudes and practices of English general 
practitioners. Alcohol and Alcoholism 1999, 34 (4) 559-566; DOI:

10.1093/alcalc/34.4.559

19. Khadjesari Z, Marston L, Petersen I, Nazareth I, Walters K. Alcohol consumption screening of newly-registered patients in primary care: a cross-sectional analysis. $\mathrm{Br}$ J Gen Pr. 2013 Oct;63(615):e706-12.

20. Babor TE, Higgins-Biddle J, Dauser D, Higgins P, Burleson JA. Alcohol screening and brief intervention in primary care settings: implementation models and predictors. J Stud Alcohol. 2005 May 1;66(3):361-8.

21. Mäkelä P, Havio M, Seppä K. Alcohol-related discussions in health care-a population view. Addiction. $2011 \mathrm{Jul}$ 1;106(7):1239-48.

22. Johansson K, Bendtsen P, Akerlind I. Early intervention for problem drinkers: readiness to participate among general practitioners and nurses in Swedish primary health care. Alcohol Alcohol. 2002 Feb;37(1).

23. Johnson M, Jackson R, Guillaume L, Meier P, Goyder E. Barriers and facilitators to implementing screening and brief intervention for alcohol misuse: a systematic review of qualitative evidence. J Public Health. 2011 Sep 1;33(3):412-21. 
Table 2: Characteristics of people with alcoholic psychosis and controls. Figures are $n(\%)$ unless otherwise stated.

\begin{tabular}{|c|c|c|c|}
\hline & $\begin{array}{l}\text { Cases } \\
\mathrm{n}=1,731\end{array}$ & $\begin{array}{l}\text { Controls } \\
n=17,310\end{array}$ & $P$ value \\
\hline \multicolumn{4}{|l|}{ Age at diagnosis(years) } \\
\hline Mean(SD) & $53.3(17.3)$ & $53.2(17.5)$ & \\
\hline \multicolumn{4}{|l|}{ Age group (n, \%) } \\
\hline $18-44$ & $595(34.4)$ & $5,999(34.7)$ & \\
\hline $45-54$ & $334(19.3)$ & $3,375(19.5)$ & \\
\hline $55-64$ & $335(19.4)$ & $3,170(18.3)$ & \\
\hline $65-74$ & $236(13.6)$ & $2,325(13.4)$ & \\
\hline$\geq 75$ & $231(13.3)$ & $2,441(14.1)$ & \\
\hline \multicolumn{4}{|l|}{ Observation time(years) } \\
\hline Median(IQR) & $5.5(2.6-9.2)$ & $4.4(2.3-8.2)$ & \\
\hline Up to 12 months & $1,731(100)$ & $17,371(100)$ & \\
\hline Up to 2years & $1,444(83.4)$ & $13,789(79.7)$ & \\
\hline Up to 5years & $912(52.7)$ & $7,779(44.9)$ & \\
\hline Up to 10 years & $362(20.9)$ & $2,901(16.8)$ & \\
\hline \multicolumn{4}{|l|}{$\operatorname{Sex}(n, \%)$} \\
\hline Male & $1,156(66.8)$ & $8,481(49.0)$ & \\
\hline Female & $575(33.2)$ & $8,829(51.0)$ & \\
\hline Smoking status & & & $<0.001$ \\
\hline Non smoker & $317(18.3)$ & $7,089(40.9)$ & \\
\hline Current smoker & $875(50.6)$ & $3,599(20.8)$ & \\
\hline Ex-smoker & $258(14.9)$ & $2,953(17.1)$ & \\
\hline Data not available & 281(16.2) & $3,669(21.2)$ & \\
\hline $\begin{array}{l}\text { Level of Alcohol } \\
\text { consumption as indicated } \\
\text { in a patient's primary care } \\
\text { record }\end{array}$ & & & $<0.001$ \\
\hline Data available & $1,369(79.1)$ & $11,326(65.4)$ & \\
\hline Never drinker & $54(3.1)$ & $1,871(10.8)$ & \\
\hline moderate drinker & $298(17.2)$ & $7,316(42.3)$ & \\
\hline Hazardous/harmful drinker & $881(50.8)$ & $1,496(8.6)$ & \\
\hline unclear & $136(7.9)$ & $643(3.7)$ & \\
\hline Data unavailable & $362(20.9)$ & $5,984(34.6)$ & \\
\hline
\end{tabular}


Table 3: Rates of primary care consultation and rate ratios comparing cases of alcoholic psychosis with the control population

\begin{tabular}{|c|c|c|c|c|c|c|c|c|c|c|c|c|}
\hline & \multirow[b]{2}{*}{ Cases } & \multirow[b]{2}{*}{ Controls } & \multirow[b]{2}{*}{ Excess } & \multirow[b]{2}{*}{ IRR $(95 \% \mathrm{Cl})$} & \multicolumn{4}{|c|}{ Males (Rate per person-years) } & \multicolumn{4}{|c|}{ Females (Rate per person-years) } \\
\hline & & & & & Cases & Controls & Excess & IRR $(95 \% \mathrm{Cl})$ & Cases & Controls & Excess & IRR $(95 \% \mathrm{Cl})$ \\
\hline $\begin{array}{l}\text { Overall } \\
\text { study } \\
\text { period }\end{array}$ & 9.9 & 6.5 & 3.4 & $1.53(1.52-1.54)$ & 9.1 & 5.2 & 3.9 & $1.76(1.74-1.78)$ & 11.5 & 7.7 & 3.8 & $1.49(1.48-1.51)$ \\
\hline \multicolumn{13}{|c|}{ Time to diagnosis(years) } \\
\hline 1 & 15.3 & 7.6 & 7.7 & $2.02(1.99-2.04)$ & 14.4 & 6.1 & 8.3 & $2.35(2.31-2.39)$ & 17.0 & 9.0 & 8.0 & $1.90(1.86-1.94)$ \\
\hline 2 & 12.2 & 7.4 & 4.8 & $1.65(1.63-1.68)$ & 11.2 & 6.0 & 5.2 & $1.86(1.82-1.89)$ & 14.2 & 8.7 & 5.5 & $1.63(1.59-1.67)$ \\
\hline 3 & 11.0 & 7.0 & 4.0 & $1.57(1.55-1.60)$ & 10.1 & 5.7 & 4.4 & $1.77(1.73-1.81)$ & 12.9 & 8.3 & 4.6 & $1.56(1.52-1.60)$ \\
\hline 4 & 10.0 & 6.6 & 3.4 & $1.51(1.48-1.54)$ & 9.2 & 5.3 & 3.9 & $1.74(1.69-1.78)$ & 11.7 & 7.9 & 3.8 & $1.47(1.43-1.52)$ \\
\hline 5 & 9.5 & 6.3 & 3.2 & $1.49(1.46-1.52)$ & 8.7 & 5.0 & 3.7 & $1.73(1.68-1.78)$ & 11.0 & 7.6 & 3.4 & $1.44(1.39-1.49)$ \\
\hline 6 & 7.9 & 6.0 & 1.9 & $1.31(1.27-1.34)$ & 6.8 & 4.8 & 2.0 & $1.43(1.38-1.48)$ & 10.1 & 7.2 & 2.9 & $1.39(1.34-1.45)$ \\
\hline 7 & 7.4 & 5.7 & 1.7 & $1.31(1.27-1.35)$ & 6.7 & 4.5 & 2.2 & $1.50(1.44-1.56)$ & 8.9 & 6.8 & 2.1 & $1.30(1.25-1.36)$ \\
\hline 8 & 6.6 & 5.4 & 1.2 & $1.22(1.18-1.26)$ & 6.1 & 4.2 & 1.9 & $1.45(1.39-1.51)$ & 7.7 & 6.6 & 1.1 & $1.17(1.11-1.23)$ \\
\hline 9 & 6.2 & 5.0 & 1.2 & $1.23(1.19-1.28)$ & 5.6 & 3.8 & 1.8 & $1.47(1.40-1.55)$ & 7.4 & 6.2 & 1.2 & $1.20(1.13-1.27)$ \\
\hline 10 & 5.3 & 4.8 & 0.5 & $1.11(1.06-1.16)$ & 4.8 & 3.6 & 1.2 & $1.37(1.29-1.45)$ & 6.3 & 5.9 & 0.4 & $1.06(0.99-1.14)$ \\
\hline
\end{tabular}


Table 4: Rates of hospitalisation and rate ratios comparing cases of alcoholic psychosis with the control population

\begin{tabular}{|c|c|c|c|c|c|c|c|c|c|c|c|c|}
\hline & \multicolumn{8}{|c|}{ Males (Rate per person-years) } & \multicolumn{4}{|c|}{ Females (Rate per person-years) } \\
\hline & Cases & Controls & Excess & $\operatorname{IRR}(95 \% \mathrm{Cl})$ & Cases & Controls & Excess & IRR $(95 \% \mathrm{Cl})$ & Cases & Controls & Excess & IRR $(95 \% \mathrm{Cl})$ \\
\hline Overall & 6.3 & 2.7 & 3.6 & $2.4(2.3-2.5)$ & 6.4 & 2.6 & 3.8 & $2.5(2.4-2.6)$ & 6.4 & 2.8 & 3.7 & $2.4(2.3-2.5)$ \\
\hline \multicolumn{13}{|c|}{$\begin{array}{l}\text { Time to } \\
\text { diagnosis(years) }\end{array}$} \\
\hline 1 & 14.4 & 3.5 & 10.9 & $4.1(3.9-4.3)$ & 14.7 & 3.3 & 11.4 & $4.5(4.2-4.8)$ & 13.9 & 3.8 & 10.1 & $3.7(3.4-4.0)$ \\
\hline 2 & 8.3 & 3.1 & 5.2 & $2.7(2.5-2.8)$ & 8.5 & 2.9 & 5.6 & $2.9(2.7-3.2)$ & 7.8 & 3.3 & 4.5 & $2.4(2.1-2.6)$ \\
\hline 3 & 6.4 & 2.9 & 3.5 & $2.2(2.0-2.3)$ & 6.5 & 2.8 & 3.7 & $2.3(2.1-2.5)$ & 6.1 & 3.1 & 3.0 & $2.0(1.8-2.3)$ \\
\hline 4 & 5.4 & 2.6 & 2.8 & 2.1(1.9-2.2) & 5.4 & 2.7 & 2.7 & $2.0(1.8-2.3)$ & 5.4 & 2.6 & 2.8 & $2.1(1.8-2.4)$ \\
\hline 5 & 4.9 & 2.4 & 2.5 & $2(1.8-2.2 .0)$ & 4.9 & 2.5 & 2.4 & $2.0(1.7-2.2)$ & 5.0 & 2.4 & 2.6 & $2.1(1.8-2.4)$ \\
\hline 6 & 4.1 & 2.3 & 1.8 & $1.8(1.6-2.0)$ & 3.6 & 2.4 & 1.2 & $1.5(1.3-1.7)$ & 5.0 & 2.2 & 2.8 & $2.3(1.9-2.7)$ \\
\hline 7 & 3.5 & 2.3 & 1.2 & $1.5(1.3-1.7)$ & 2.8 & 2.6 & 0.2 & $1.1(0.9-1.3)$ & 4.8 & 2.1 & 2.7 & $2.3(1.9-2.8)$ \\
\hline 8 & 3.3 & 2.1 & 1.2 & $1.6(1.3-1.8)$ & 2.6 & 2.3 & 0.3 & $1.1(0.9-1.4)$ & 4.8 & 1.9 & 2.9 & $2.5(2.0-3.1)$ \\
\hline 9 & 2.6 & 2.0 & 0.6 & $1.3(1.1-1.6)$ & 2.5 & 1.9 & 0.6 & $1.3(1.0-1.6)$ & 2.8 & 2.0 & 0.8 & $1.4(1.0-1.9)$ \\
\hline 10 & 2.3 & 1.6 & 0.7 & $1.4(1.1-1.7)$ & 2.3 & 1.6 & 0.7 & $1.5(1.1-1.9)$ & 2.2 & 1.7 & 0.5 & $1.3(0.9-1.9)$ \\
\hline
\end{tabular}


Table 5: Prevalence of alcohol attributable comorbidities

\begin{tabular}{|c|c|c|c|c|}
\hline & $\begin{array}{l}\text { Cases } \\
(n=1,731)\end{array}$ & $\begin{array}{r}\text { Controls } \\
(n=17,310)\end{array}$ & $\begin{array}{l}\text { OR (95\% Cl) } \\
\text { Model 1* }\end{array}$ & $\begin{array}{l}\text { OR (95\% Cl) } \\
\text { Model 2** }\end{array}$ \\
\hline & $n(\%)$ & $n(\%)$ & & \\
\hline \multicolumn{5}{|l|}{ Overall numbers presenting with: } \\
\hline Any alcohol attributable disease & $1,157(66.8)$ & $574(33.2)$ & & \\
\hline At least one alcohol-specific disease & $354(20.5)$ & $75(0.4)$ & & \\
\hline At least one alcohol-related disease & $1,104(63.8)$ & $5,376(31.1)$ & $3.9(3.5-4.3)$ & \\
\hline Malignant neoplasms & $27(1.6)$ & $230(1.3)$ & $1.2(0.8-1.8)$ & $1.4(0.9-2.1)$ \\
\hline Diabetes & $127(7.3)$ & $923(5.3)$ & $1.4(1.2-1.7)$ & $1.3(1.1-1.7)$ \\
\hline Epileptic seizures & $134(7.7)$ & $201(1.2)$ & $7.1(5.7-8.9)$ & $7.1(5.6-9.0)$ \\
\hline Cardiovascular diseases & $396(22.9)$ & $2,697(15.6)$ & $1.6(1.4-1.8)$ & $1.7(1.5-1.9)$ \\
\hline Digestive diseases & $72(4.2)$ & $253(1.5)$ & $2.9(2.2-3.8)$ & $3.2(2.4-4.2)$ \\
\hline Unintentional injuries & $712(41.1)$ & $2,381(13.8)$ & $4.4(3.9-4.9)$ & $4.5(4.0-5.0)$ \\
\hline Intentional injury/self-harm & $326(18.8)$ & $290(1.7)$ & $13.6(11.5-16.1)$ & $11.8(9.8-14.1)$ \\
\hline
\end{tabular}


Table 6: Proportion and OR $(95 \% \mathrm{Cls})$ of patients with alcohol use records stratified by sex and age

\begin{tabular}{|c|c|c|c|c|c|c|c|c|c|}
\hline & Overall & & & Males & & & Females & & \\
\hline & Cases & Controls & OR $(95 \% \mathrm{Cl})$ & Cases & Controls & OR (95\% Cl) & Cases & Controls & OR $(95 \% \mathrm{Cl})$ \\
\hline & $\mathrm{n}=1,731$ & $\mathrm{n}=17,310$ & & $\mathrm{n}=1,156$ & $\mathrm{n}=8,481$ & & $n=575$ & $n=8,829$ & \\
\hline Overall & $1,369(79.1)$ & $11,326(65.4)$ & $1.5(1.3-1.7)^{\mathrm{a}}$ & $928(80.3)$ & $5,307(62.6)$ & $1.7(1.5-2.0)^{\mathrm{b}}$ & $441(76.7)$ & $6,019(68.2)$ & $1.2(0.9-1.5)^{b}$ \\
\hline \multicolumn{10}{|c|}{ Age at diagnosis } \\
\hline $18-44$ & $437(73.4)$ & $3718(61.9)$ & $1.8(1.5-2.3)$ & $307(74.3)$ & 1704(55.9) & $2.3(1.8-2.8)$ & $130(71.4)$ & 2014(68.3) & $1.2(0.9-1.7)$ \\
\hline $45-54$ & $274(82.0)$ & 2239(66.3) & $2.5(1.9-3.3)$ & $181(81.9)$ & $1112(64.1)$ & $2.6(1.9-3.6)$ & $93(82.3)$ & 1127(68.8) & $2.1(1.4-3.4)$ \\
\hline $55-64$ & $287(85.7)$ & $2137(67.4)$ & $2.9(2.2-4.2)$ & $206(86.2)$ & 1051(65.6) & $3.5(2.3-5.2)$ & $81(84.3)$ & 1086(69.3) & $2.3(1.2-4.2)$ \\
\hline $65-74$ & $188(79.7)$ & $1608(69.2)$ & $1.7(1.2-2.5)$ & $125(83.3)$ & $768(67.5)$ & $2.2(1.4-3.5)$ & $63(73.3)$ & $840(70.8)$ & $1.2(0.7-2.1)$ \\
\hline$\geq 75$ & 183(79.2) & $1624(66.5)$ & $1.9(1.3-2.7)$ & $109(82.0)$ & $672(70.4)$ & $2.1(1.3-3.4)$ & $74(75.5)$ & $952(64.0)$ & $1.6(1.0-2.7)$ \\
\hline
\end{tabular}

aOR adjusted for age, sex and consulting rate of patients

bOR adjusted for age and consulting rate of patients

Irtest for sex interaction: 0.0012; test for age interaction: 0.12 
Table 7: Proportion and OR (95\% Cls) of patients with any alcohol intervention record, stratified by age.

\begin{tabular}{llll}
\hline & Cases & Controls & OR $(\mathbf{9 5 \%} \mathbf{C l})^{\mathbf{a}}$ \\
\hline $\begin{array}{l}\text { Overall } \\
\text { Age at diagnosis }\end{array}$ & $\mathrm{n}=1,731$ & $\mathrm{n}=17,310$ & \\
$\mathbf{1 8 - 4 4}$ & & $908(5.3)$ & $3.7(2.9-4.6)^{\mathrm{b}}$ \\
$\mathbf{4 5 - 5 4}$ & $109(18.3)$ & $228(3.8)$ & $4.3(3.1-5.8)$ \\
$\mathbf{5 5 - 6 4}$ & $76(22.8)$ & $198(5.9)$ & $3.2(2.2-4.8)$ \\
$\mathbf{6 5 - 7 4}$ & $72(21.5)$ & $185(5.8)$ & $3.7(2.7-5.2)$ \\
$\mathbf{2 7 5}$ & $63(26.7)$ & $167(7.2)$ & $3.8(2.7-5.2)$ \\
\hline aOR adjusted for sex and consulting rate unless otherwise stated badjusted for age, sex and \\
Consulting rate; LRT for sex interaction P value: 0.06; LRT for age interaction P value 0.02 \\
\hline
\end{tabular}


Figure 1: Odds ratios for the association between each morbidity and alcoholic psychosis versus the control population at each time period, adjusted for age, sex and smoking status. Point estimates are shown in the data label below.

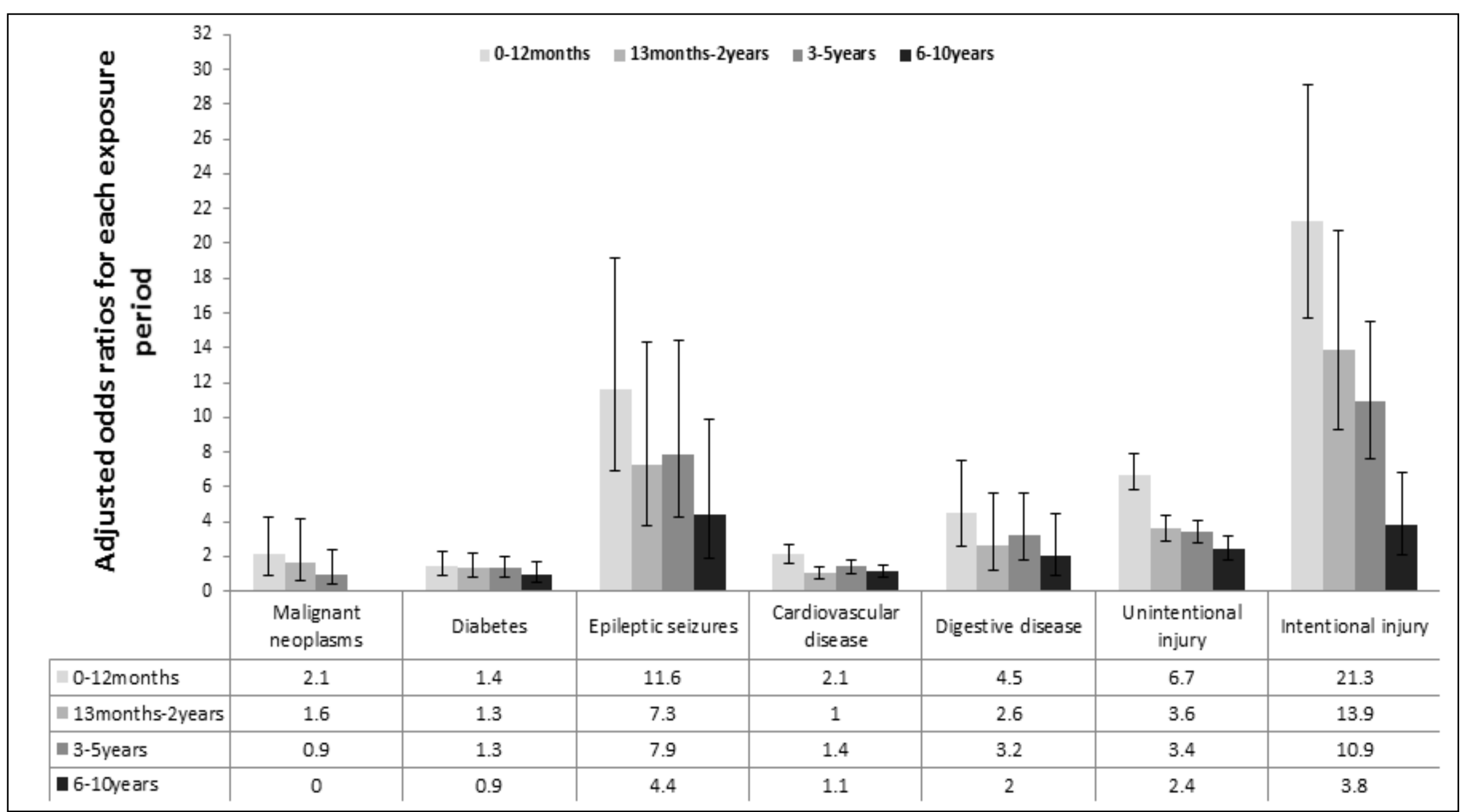

